die Beschuldigten die Aufforderung der Ast. v. 15.7.2008 bis zum 31.12.2008 durch Abgabe einer Kündigungserklärung gegenüber der $m$ hätten umsetzen müssen. Eine solche Kündigung wäre erst zum 31.12.2009 wirksam geworden; das berufsgerichtliche Verfahren ist jedoch schon zum 30.1.2009 eingeleitet worden. Überdies haben die Beschuldigten im Laufe des Jahres 2009 das Gesellschaftsverhältnis ohnehin beendet.
Eine außerordentliche Kündigung wäre den Beschuldigten schon deswegen nicht zuzumuten gewesen, weil nach den Umständen des konkreten Falls nicht geklärt gewesen ist, ob ihre Beteiligung tatsächlich gegen berufsrechtliche Vorschriften verstieß, und es auch der Ast. im Rahmen des berufsgerichtlichen Verfahrens im Kern um die Klärung der objektiven Rechtslage, der Zulässigkeit einer gesellschaftsrechtlichen Beteiligung an der $m$ ging.

REZENSIONEN

DOI: 10.1007/s00350-011-2964-6

\section{Rechtsfragen des Einsatzes der Telemedizin im Rettungsdienst. Eine Untersuchung am Beispiel des Forschungsprojektes Med-on-@ix.}

Von Christian Katzenmeier und Stefania Schrag-Slavu. Verlag Springer, Berlin und Heidelberg 2010, XI u. 188 S., geb., $€ 79,95$

Das in Band 2 der Kölner Schriftenreihe zum Medizinrecht erschienene Werk geht medizinrechtlichen Fragestellungen des Einsatzes der Telemedizin im Rettungsdienst nach und stellt damit einen wichtigen Zukunftsmarkt für den Einsatz der Telemedizin vor.

Das Buch basiert auf einem Rechtsgutachten, das die Autoren im Rahmen des Forschungsprojektes Med-on-@ix, einem System zur Verbesserung der Notfallversorgung, erstellt haben, und bietet dem Leser einen umfassenden Überblick zur Struktur sowie den rechtlichen Aspekten der Notfallmedizin und schließlich zu den relevanten medizin-, haftungs- und datenschutzrechtlichen Fragestellungen des Projekts. Darüber hinaus erfolgt eine vergleichende Betrachtung ausgewählter europäischer Rettungsdienstsysteme.

Das begutachtete Projekt beschäftigt sich mit der deutschlandweiten Einführung moderner Informations- und Kommunikationstechnologien bei allen teilnehmenden Partnern des Rettungsdienstes, sei es Rettungsleitstelle, Rettungsdienstpersonal, Notarzt oder Krankenhaus. Im Mittelpunkt des Systems steht der Aufbau eines Kompetenzzentrums, das mit hochqualifizierten und erfahrenen Notfallmedizinern besetzt werden soll. Den Notärzten am Einsatzort würde somit eine additive Beratung durch die Notfallmediziner aus dem Kompetenzzentrum, sog. „Telenotärzte“, zur Seite gestellt werden. Im Wege der telemetrischen Datenübertragung sollen einsatz- und patientenbezogene Daten, wie z.B. EKG, Vitalparameter oder Videofrequenzen und Bildmaterial in Echtzeit zwischen dem vor Ort tätigen ärztlichen sowie nichtärztlichen Rettungsdienstpersonal und den Telenotärzten im Kompetenzzentrum übermittelt werden können.

Obwohl der deutsche Rettungsdienst im internationalen Vergleich eine Spitzenstellung einnimmt, so die Verfasser, bestünde die Gefahr, dass der steigende Bedarf an Notärzten vor allem in strukturschwachen Regionen in absehbarer Zeit nicht mehr gedeckt werden könne. Mit Hilfe der Telemedizin ließen sich die wenigen Notärzte auf die wirklich relevanten Einsätze beschränken und damit der Notarztmangel kompensieren und zugleich die Kosten des Rettungsdienstsystems beträchtlich senken. Das System zielt darauf ab, die Qualität der Notversorgung und deren Effizienz bei gleichzeitiger Kostenreduktion zu steigern.

Nach einer ausführlichen Einführung in das deutsche Rettungswesen, seine Organisation und Struktur, nehmen sich die Autoren der rechtlichen Kernfragen an. Die (medizin-)rechtliche Durchführbarkeit des Einsatzes der Telemedizin im Rettungsdienst und die damit verbundenen technischen und insbesondere datenschutzrechtlichen Anforderungen bestimmen die Teile 2 bis 4 des Buches. Dabei erfährt das im telemedizinischen Kontext oftmals vernachlässigte, aber für die Praxis so wichtige Medizinprodukterecht eine besondere Beachtung (Teil 3). Es werden Definitions- und Abgren-

Rechtsanwalt Prof. Dr. med. Dr. iur. Christian Dierks, Berlin, Deutschland zungsfragen aufgegriffen sowie Rechte und Pflichten der Anwender herausgearbeitet, die sich insbesondere aus der Kombination der zur Informationsübertragung erforderlichen Nicht-Medizinprodukte, wie Ethernetkabel, DSL-Modem und ISDN-Anschluss mit den zur Informationsgewinnung eingesetzten Medizinprodukten (z. B. EKG, elektronisches Stethoskop) ergeben. Im 5. Teil des Bandes wird das Rettungswesen in Europa vergleichend gegenübergestellt, um die Möglichkeiten sowie die Sinnhaftigkeit einer Implementierung des Med-on-@ix-Systems in anderen europäischen Ländern zu eruieren, bevor schließlich in Teil 6 die wesentlichen Ergebnisse des Forschungsprojektes zum Einsatz der Telemedizin im Rettungsdienst zusammengefasst werden. Gerne hätte der Leser nach der rein rechtlichen Lektüre noch einen kurzen Überblick über die bislang gesammelten Ergebnisse und Daten zur praktischen Umsetzung sowie über die Einsparung von Kosten und Ressourcen erhalten. So aber bleiben die Verfasser der Überschrift ihres Buches treu und beschränken ihre Ausführungen zum Forschungsprojekt auf die Rechtsfragen. Für eine Folgeauflage wäre daher die ergänzende Veranschaulichung der praktischen Relevanz mit Daten zu erwägen.

In den letzten Jahren hat sich in Deutschland eine vielfältige und kaum noch überschaubare Forschungs- und Entwicklungsaktivität zur Telemedizin entwickelt. Es ist den Verfassern gelungen, ein für Notfallpatienten, Rettungssanitäter, aber vor allem auch Anbieter telemedizinischer Produkte gleichermaßen hochinteressantes Einsatzgebiet der Telemedizin vorzustellen und rechtlich abschließend aufzuarbeiten. Die Telemedizin ermöglicht eine zeitnahe und qualifizierte Patientenbehandlung bei gleichzeitiger Schonung von Ressourcen, so die Verfasser. Möge ihr daher der Sprung von der Forschung in die breite Anwendung gelingen!

\section{Arbeitsrecht im Krankenhaus.}

Herausgegeben von Stephan Weth, Heike Thomae und Hermann Reichold. Verlag Dr. Otto Schmidt, 2. Aufl. Köln 2011, 1034 S., gbd., $€ 99,00$

Vier Jahre nach seinem erstmaligen Erscheinen liegt nunmehr das aktualisierte Mammutwerk zum Krankenhausarbeitsrecht in einer neu bearbeiteten Auflage vor. Mit einem Textteil von 935 Seiten handelt es sich wohl um die umfassendste Aufarbeitung von arbeitsrechtlichen Fragestellungen im Zusammenhang mit Krankenhausbeschäftigten. Erklärtes Ziel des von Weth, Thomae und Reichold herausgegebenen Werkes ist es, „,der Praxis ein taugliches Hilfsmittel an die Hand zu geben und allen, die im Krankenhaus mit arbeitsrechtlichen Fallgestaltungen konfrontiert sind, Hilfestellungen zu geben, um Probleme aufzuspüren, Fallen zu vermeiden und Lösungen zu finden“. Insgesamt zeichnen acht namhafte Autoren aus Wissenschaft, Rechtsprechung und Anwaltschaft für den Inhalt verantwortlich. Trotz seiner Untergliederung in 13 Teile lassen sich vier Hauptteile des Werkes ausmachen. Zunächst werden auf den ersten 110 Seiten die krankenhausrechtlichen Grundlagen erläutert. Hier geht es etwa

Prof. Dr. iur. Oliver Ricken, Universität Bielefeld, Deutschland 